Bol. Acad. peru. leng. 47. 2009 (55-79)

\title{
ESTUDIO LEXICOGRÁFICO DE LA OBRA LOS INOCENTES, DE OSWALDO REYNOSO
}

\section{ÉTUDE LEXICOGRAPHIQUE DU ROMAN LOS INOCENTES D' OSWALDO REYNOSO}

\author{
A LEXICOGRAPHIC STUDY OF REYNOSO'S NOVEL \\ LOS INOCENTES \\ Luisa Portilla Durand \\ Universidad Nacional Mayor de San Marcos
}

Resumen:

El artículo presenta el estudio lexicográfico de un corpus constituido por 47 voces propias del léxico peruano puestas de manifiesto en la primera obra en prosa del escritor peruano Oswaldo Reynoso: Los inocentes (1961). El número de voces seleccionadas responde a que este año (2008) se cumplen 47 años de publicación de Los inocentes. Por otra parte, el objetivo del presente estudio es mostrar la riqueza léxica de esta obra y, asimismo, reconocer el aporte de Oswaldo Reynoso al rescate de voces propias del habla peruana, sobre todo del léxico coloquial y popular, lo que se constituye en una importante fuente de vitalización para nuestra lengua.

Résumé:

L'article présente l'étude lexicographique d'un corpus constitué de 47 mots propres au lexique péruvien mis en exergue dans le premier roman de l'écrivain péruvien Oswaldo Reynoso: Los inocentes (1961). Le nombre de mots sélectionnés correspond au $47^{\mathrm{e}}$ anniversaire de la première publication de Los inocentes. De plus, l'objectif de cette étude est de découvrir la richesse lexicale de cette œuvre et donc, de reconnaître 
l'apport d'Oswaldo Reynoso qui vient au secours de mots propres à la langue péruvienne, en particulier le lexique colloquial et populaire, représentant une importante source de vitalisation de notre langue.

Abstract:

Selected to match the 47 years of the publication of Reynoso's Los Inocentes, a similar number of terms belonging to Peruvian lexical lore are studied in this article, to show the richness of popular and colloquial language which Reynoso contributes to enhance so as to become an important source for the vitalization of Peruvian Spanish.

Palabras clave:

Oswaldo Reynoso; lexicografía; léxico peruano.

Mots clés:

Oswaldo Reynoso; étude lexicographique; lexique péruvien; colloquial, populaire.

Key words:

Oswaldo Reynoso; Lexicography; Peruvian speech.

El presente trabajo constituye parte del estudio lexicográfico de la primera obra en prosa del escritor peruano Oswaldo Reynoso: Los inocentes (1961). ${ }^{1} \mathrm{Al}$ respecto, lo que pretendo es presentar una muestra -sólo una muestra- de la riqueza léxica de esta obra y, asimismo, reconocer el aporte del autor al rescate del léxico peruano, sobre todo en el nivel de uso coloquial y popular. ${ }^{2}$

$1 \quad$ La primera publicación de Los inocentes (1961) estuvo a cargo de Ediciones La Rama Florida, como parte de la colección de Narradores Peruanos Contemporáneos, dirigida por Wáshington Delgado.

2 Esta clasificación la propone Luis Hernán Ramírez (1996) en "Niveles de uso lingüístico". 
Para llevar adelante este estudio, he revisado las tres últimas ediciones del DRAE: 1984, 1992 y 2001. Así, luego de leer Los inocentes, de Oswaldo Reynoso, organicé un corpus básico de 47 palabras ${ }^{3}$ que por su uso difundido esperaba encontrar en los diccionarios antes mencionados. Como resultado de la búsqueda, obtuve lo siguiente: de las 47 palabras seleccionadas, sólo una había sido incluida en el DRAE 84 -después de 23 años de haberse publicado Los inocentes- como parte de una forma compleja: ${ }^{4}$ en pelo (no tener pelos en la lengua). (Véase NUEVA FORMA COMPLEJA INCLUIDA EN EL DRAE 84). A los 31 años de publicada la obra, encontré en el DRAE 92 cinco inclusiones más: me refiero al agregado de una acepción en el caso de las palabras acostar, corrido, espeso y pilcha, y de una forma compleja en mosca (por si las moscas). (Véase FORMA COMPLEJA Y NUEVAS ACEPCIONES INCLUIDAS EN EL DRAE 92). Recién a los 40 años de publicación de Los inocentes aparecen en el DRAE 2001, como forma compleja, entrada ${ }^{5}$ o acepción nueva, nueve inclusiones más: arrochar, asado, campana, chapa, oreja (parar la oreja), revolcar, sobrado, teclo, viejo. (Véase FORMA COMPLEJA Y NUEVAS ENTRADAS Y ACEPCIONES INCLUIDAS EN EL DRAE 2001). Sin embargo, la lista de 47 palabras que seleccioné no se agotaba aún, así que seguí revisando el DRAE 2001 con la intención de hallar, con la marca de otros países, los usos que se ponen de manifiesto en la obra de Oswaldo Reynoso. La búsqueda resultó provechosa, ya que me permitió encontrar 23 palabras más del corpus: bacán, bronca, cebada, chicoco, collera, cuera, desgraciar (desgraciarse), gil, lechero, mandar (mandar a pasear), mocoso, paleteo, peluca, pendejo, polilla, punto (tomar como punto), sabido, sonar, sufrido, taba, templado, tono, voz (pasar la voz), las cuales coinciden en sus acepciones, o por lo menos en la entrada, con formas

3 Opté por seleccionar 47 palabras del corpus de Los inocentes por cumplirse, a la fecha (2008), 47 años de la primera publicación de la obra.

$4 \quad$ Según se lee en las "Advertencias para el uso" en el DRAE 2001 (p. xL), las formas complejas son "aquellas series de palabras que, combinadas de una determinada manera, expresan conceptos no interpretables mediante la simple adición de los significados de sus componentes (de perlas, aceite virgen, no ganar para sustos)". Cuando éstas aparecen, se ubican en la parte final del artículo lexicográfico, antecedidas por doble barra gruesa.

5 Palabra, locución, frase, sintagma, signo o conjunto de letras o signos que encabeza un artículo de diccionario, vocabulario, glosario, terminología, índice, ficha, etc., y que es objeto de definición o explicación y, eventualmente, de tratamiento enciclopédico. (Martinez de Sousa, 1995: 180). 
usadas en otros países de habla hispana. (Véase ENMENDADURAS PROPUESTAS AL DRAE 2001). Restaban entonces nueve palabras de las seleccionadas del corpus: capazote, cargamontón (hacer cargamontón), enchatar, ensarte, full, planero, tombo, trome, zafa, las cuales no aparecen como entrada o como acepción en el DRAE 2001, y que, sin embargo, a la fecha (2008), tienen 47 años de vigencia, por lo que las he considerado como parte de las NUEVAS ENTRADAS Y ACEPCIONES PROPUESTAS AL DRAE.

Señalaré a continuación lo que el lector encontrará en las páginas siguientes, no sin antes indicar que las abreviaturas usadas en este trabajo así como los signos empleados son los mismos que se utilizan en el DRAE 2001.

a) Se muestran los hallazgos que hice en las tres últimas ediciones del DRAE (1984, 1992 y 2001) en relación con las 47 palabras que forman parte del corpus seleccionado.

b) Se presentan, asimismo, 22 propuestas de enmendadura al DRAE 2001, en las que se consideran, fundamentalmente, la adición de la marca diatópica Perú así como el agregado de acepciones en las entradas citadas.

c) Se propone también el ingreso de nueve entradas al DRAE, con sus correspondientes acepciones.

d) Por otra parte, acompañando a cada entrada y definición, se muestra el respaldo escrito, es decir, las citas de la obra Los inocentes, que me han permitido avalar el uso de las 47 palabras que han sido motivo de estudio en esta investigación.

Finalmente, presento las conclusiones a las que he arribado, así como las REFERENCIAS BIBLIOGRÁFICAS que me han sido útiles para llevar adelante este trabajo. 
Nueva forma compleja incluida en el DRAE 84

pelo. $[\ldots] \|$ no tener uno pelos en la lengua. fr. fig. fam. no tener frenillo en la lengua. [...]

$\mathrm{Al}$ revisar la remisión hallé:

frenillo. [...]\|no tener uno frenillo, o no tener uno frenillo en la lengua. fr. fig. fam. Decir sin reparo ni empacho lo que piensa o siente, o hablar con demasiada libertad y desembarazo. [...]

En el DRAE 1992, encontré lo siguiente:

pelo. [...] $\|$ no tener uno pelos en la lengua. fr. fig. y fam. Decir sin reparo ni empacho lo se que piensa o siente, o hablar con demasiada libertad y desembarazo.

En el DRAE 2001 se mantiene la definición anterior, pero varían las marcas que acompañan a la forma compleja: ya no aparece la marca fig. ('figurado') y se cambia fam. ('familiar') por coloq. ('coloquial'):

pelo. $[. .$.$] \| no tener alguien pelos en la lengua. fr. coloq. Decir sin$ reparo ni empacho lo que piensa o siente, o hablar con demasiada libertad y desembarazo. [...]

Ejemplo de uso:

-Más respeto. No hable de esa forma de mi compadre.

-No, don Lucho, yo no tengo pelos en la lengua. Yo siempre, siempre digo lo que pienso, lo-que-pien-so-y-na-da-más. (41)

Forma compleja y nuevas acepciones incluidas en el DRAE 92

acostar. [...]\| 8. prnl. Mantener relación sexual una persona con otra. Ú. con la prep. con. 
En el DRAE 2001, la definición se presenta de la siguiente manera:

acostar. [...]\| 7. prnl. Dicho de una persona: Mantener relación sexual con otra. No quiere acostarse CON nadie. [...]

Como puede observarse, se ha aislado el contorno ${ }^{6}$ (aunque éste corresponde a un adjetivo y no a un verbo: debería aparecer Referido a una persona en vez de "Dicho de una persona") y se ha agregado un ejemplo a la definición.

\section{Ejemplo de uso:}

Voy a estar solo en mi hueco y he quedado con la gila* para acostarnos en mi cama: vamos a estar solititísimos. (73)

${ }^{*}$ Ver definición en gil ${ }^{2}$, la.

$* * * *$

corrido, da. [...] adj. [...] $\mid$ 6. fam. Aplícase a la persona de mundo, experimentada, astuta. [...]

En el DRAE 2001, hallamos lo siguiente:

corrido, da. [...] adj. [...]\|4 4. coloq. Dicho de una persona: De mundo, experimentada y astuta. [...]

En primer lugar, en el DRAE 2001 se ha cambiado la marca fam. ('familiar') por coloq. ('coloquial'); en segundo lugar, se ha aislado el contorno; por otra parte, no coincido en la forma como ha sido propuesta la definición, por lo que propongo la siguiente:

corrido, da. [...] adj. [...]\| coloq. Perú. Dicho de una persona: Experimentada o de mundo.

6 Conjunto de elementos de información no esencial añadidos a una definición (Martínez de Sousa, 1995: 68). 
Ejemplos de uso:

-Usted que es corrido sabe que del plan de paleteo* y chupete hay que pasar a otra cosa, uno no puede quedarse en el plan de cochineo. $(72-73)$

*Ver definición en paleteo.

Por eso, para que el patrullero no se lo cargue, tiene que poner cara de "maldito", de hombre "corrido", torcer los ojos, fumar como vicioso, hablar groserías, fuerte, para que lo escuchen, caminar a lo James Dean, es decir, como cansado de todo, y con las manos en los bolsillos y, de vez en cuando, toser ronco, profundo. (86-87)

espeso, sa. [...] adj. [...]\|6. fig. Ar., Perú y Venez. Pesado, impertinente, molesto.

En el DRAE 2001, la definición se presenta de la siguiente manera:

espeso, sa. [...] adj. [...]\|4. Ar., Perú y Ven. Pesado, impertinente, molesto. [...]

Obsérvese, en primer lugar, que en el DRAE 2001 ya no aparece la marca fig. 'figurado'; en segundo lugar, la definición, basada en un listado sinonímico, es incorrecta, además de redundante, pues basta con remitir a impertinente para definir espeso, por lo que propongo lo siguiente:

espeso, sa. [...] adj. [...] \|coloq. Perú. impertinente ( $\mid$ que molesta de palabra o de obra).

Ejemplo de uso:

-Yayayayayaaa, calla, calla. Zafazafazafazafa. Eres más espeso. Deja que cuente. Está bonito. Así fuera mentira, qué importa - protestó Natkinkón. (46) 
Ver también ejemplo de uso en asado, da.

$* * * *$

mosca. [...] $\|$ por si las moscas. fr. fig. y fam. Por si acaso, por lo que pueda suceder. [...]

En el DRAE 2001, se halla lo siguiente:

mosca. [...]\| por si las s. loc. adv. coloq. Por si acaso, por lo que pueda suceder. [...]

En la definición, por lo que pueda suceder es redundante, pues equivale a por si acaso ('en previsión de una contingencia').

Obsérvese, por otra parte, que no aparece la marca fig. 'figurado', y que se ha cambiado fr. ('frase') por loc. adv. ('locución adverbial') y fam. ('familiar') por coloq. ('coloquial').

Ejemplo de uso:

-Lo tienes que hacer con cuidado. Por si las moscas, compra en la botica algodón, gasa, alcohol. (74)

$* * * *$

pilcha. [...] f. [...]\|3. Argent. Prenda de vestir, particularmente si es elegante y cara. Ú. m. en pl.

En el DRAE 2001, se halla lo siguiente:

pilcha. $\quad[\ldots]$ f. $[\ldots] \| 3$. coloq. Arg., Perú y Ur. Prenda de vestir, particularmente si es elegante y cara. Ú. m. en pl.

Al respecto debo señalar, en primer lugar, que la definición es impropia, pues incluye contorno: particularmente si es elegante y cara; en segundo lugar, en el Perú, esta palabra se usa, más bien, en singular. Por lo dicho, propongo lo siguiente: 
pilcha. [...]\| coloq. Perú. Generalmente si es elegante y cara: Prenda de vestir.

\section{Ejemplo de uso:}

Esta noche no podrá decir que no. Estará alegre. Es su cumpleaños. Y estoy bien firme. Mi peluca* está recortada. No hay caso, Manos Voladoras: un artista. Mis zapatos, de gamuza. Estreno pilcha azul y corbata de seda italiana bien bacán. (80)

${ }^{*}$ Ver definición en peluca y bacán ${ }^{1}$, respectivamente.

Forma compleja y nuevas entradas y acepciones incluidas en el DRAE 2001

arrochar. tr. coloq. Perú. Rechazar, despreciar.

El uso de la coma en la definición es ambiguo, por otra parte, el orden de las palabras propuestas como sinónimos debe invertirse y obviarse la segunda, por lo que propongo la siguiente enmienda:

arrochar. tr. coloq. Perú. despreciar ( $\|$ desestimar y tener en poco).

Ejemplo de uso:

Juanita, ahora, estás muy cambiada. Pero yo sé que sólo es cáscara. Estoy seguro que basta una palabra mía para que seas la chicoquita* de quince años. Ahora, siempre me arrochas. (83)

*Ver definición en chicoco, ca.

$* * * *$

asado, da. [...] ||adj. Perú. Enojado, enfadado. [...]

En la definición, enfadado es redundante; pues en el DRAE, enfadar se define como 'causar enfado', y enfado es -según el mismo DRAEenojo 'movimiento del ánimo que suscita ira'; por ello, está de más incluir enfadado en la definición, por lo que propongo la siguiente enmienda: 
asado, da. [...]\|adj. Perú. Enojado. [...]

Ejemplo de uso:

-Yo también he salido en los comercios, ¿recuerdan? Apenas tenía catorce años y ya estaba aburrido de mi casa: todos los días había correa. Y el espeso* del Borrao, ese de Normas Educativas, me llevaba bronca*, me tenía asado. (45) *Ver definición en espeso y bronca (llevar bronca), respectivamente. $* * * *$

campana. [...]\|6. m. Ladrón que permanece fuera del lugar del robo para alertar a sus cómplices. Huyeron a tiempo porque les avisó el campana. [...]

Ejemplo de uso:

-Ese muchachito promete. A ver, don Lucho, dos pomos, por favor. Hay que tener cojones para asaltar y robar un For, solito, sin ayuda, sin campana. (49)

$* * * *$

chapa. [...] || 15. f. Perú. apodo ( || nombre que suele darse a una persona). [...]

Ejemplo de uso:

In-dis-cu-ti-ble-men-te-e-res-un-Prín-ci-pe. To-do-un-Prín-ci-pe. Y desde ese día se le metió en la cabeza que yo era un Príncipe. Porque Lima, siendo Ciudad de los Reyes, tenía que tener un Príncipe. Y me quedé con la chapa. (54-55)

$* * * *$

oreja. [...] $\|$ parar la . fr. coloq. Am. Mer., Guat. y Méx. aguzar las orejas ( $\|$ prestar atención). [...] 
Ejemplo de uso:

De repente, el señor se despertó y, al no encontrar su cartapacio, armó tal bulla que el chofer tuvo que parar el vehículo. Pero al encontrarlo en el suelo se alegró y, en alta voz, dijo que ahí llevaba más de diez mil soles. Sorprendido, paré la oreja. (55-56)

revolcar. [...]|| 5. prnl. vulg. Practicar juegos eróticos o mantener relaciones sexuales. [...]

Ejemplo de uso:

Y como su casa es estrecha y su hijo duerme en el mismo cuarto y es un estorbo para sus aventuritas, lo manda al taller y mientras mi pobre Príncipe tirita el viejo sucio se revuelca calientito con alguna polilla* cochina. (42)

*Ver definición en polilla.

$* * * *$

sobrado, da. [...] adj. [...] ||4. coloq. Ecuad. y Perú. creído ( || muy pagado de sí mismo). [...]

Ejemplo de uso:

Míralo, a pesar de ser un poco gordo y casi teclo*, cómo se desliza suavecito alrededor de la mesa. Y cómo pica a los sobrados. (66)

*Ver definición en teclo. $* * * *$

teclo, cla. m. y f. coloq. Perú. anciano (\| persona de mucha edad).

Ejemplo de uso:

Bol. Acad. peru. leng. 47(47), 2009 
Mañana domingo, o sea hoy, mis teclos se van a Chosica, no voy con ellos: les he dicho que tengo que estudiar para los exámenes. (p73) Ver también ejemplo de uso en sobrado, da.

$* * * *$

viejo, ja. [...] || 8. m. y f. coloq. Am. padres (

Ejemplos de uso:

El único que hace lo que le da la gana es Colorete. Grita y se impone y, si el viejo protesta, le saca en cara su negocio, su cantar: el viejo, su viejo, es cabrón. (24-25)

Cuando el viejo me vio se puso alegre y me abrazó. Mi viejita lloró y en la noche preparó arroz con pato. (46)

Ver también ejemplo de uso en full (a todo ).

\section{Enmendaduras propuestas al DRAE 2001}

bacán ${ }^{1}$, na. adj. Chile, Col. y Cuba. En lenguaje juvenil, muy bueno, estupendo, excelente. [...]

En primer lugar, en el Perú no se reconoce la forma bacana; en segundo lugar, bacán no sólo se usa en el lenguaje juvenil; asimismo, es incorrecto el listado sinonímico como parte de la definición, por lo que, basándome en el corpus seleccionado, propongo lo siguiente:

bacán ${ }^{1}$, na. [...] $\|$ adj. Perú. excelente ( $\|$ que sobresale en mérito o estimación). [...]

Ejemplo de uso:

Esta noche no podrá decir que no. Estará alegre. Es su cumpleaños. Y estoy bien firme. Mi peluca* está recortada. No hay caso, Manos 
Voladoras: un artista. Mis zapatos, de gamuza. Estreno pilcha* azul y corbata de seda italiana bien bacán. (80)

*Ver definición en peluca y pilcha, respectivamente. $* * * *$

bronco, ca. $[. .$.$] \| tener bronca a alguien. fr. coloq. Am. fig. tener$ entre ojos.

En el DRAE 92, el artículo ${ }^{7}$ figuraba de la siguiente manera:

bronca. [...] $\|$ tener bronca a alguien. fr. fig. y fam. Amér. tener entre ojos.

Al comparar lo hallado en las dos últimas ediciones del DRAE, llama la atención, además del cambio de ubicación, la presencia de la marca fig. 'figurado', que en las definiciones que se han tratado anteriormente había desaparecido: ¿qué diferencia establece? Ramón Trujillo (2005), al observar que en el DRAE 2001 no aparecían los usos figurados tan frecuentes en las ediciones anteriores, comenta lo siguiente: “CCómo se llegó a la conclusión de que la distinción entre lo de recto y lo de figurado no conduce a ninguna parte? ¿No sabían los autores del Diccionario que no existían en realidad significados rectos y figurados como "objetos" diferentes, o, mirado desde otro ángulo, que es siempre recta la forma de cada palabra, si se separa de sus usos o variantes, y que es siempre figurada cada variante particular, cada percepción individual de una palabra en una circunstancia cualquiera?" Por otra parte, la remisión es incorrecta, pues la definición de la forma compleja se halla en traer entre ojos y no en tener entre ojos.

Por lo dicho, y ya que en el Perú se usa, además, otra forma compleja con la misma acepción, propongo lo siguiente:

bronco, ca. [...] || llevar bronca a alguien. fr. coloq. Perú. traer entre

$7 \quad$ Parte de un diccionario, glosario o vocabulario encabezada por una unidad léxica y cuya finalidad es definirla o compararla con otra u otras. (Martínez de Sousa, 1995: 41). La unidad léxica es el "objeto de definición de un diccionario, glosario, vocabulario, etc." (ibídem, p. 342).

Bol. Acad. peru. leng. 47(47), 2009 
ojos ( $\|$ tenerle mala voluntad). $\|$ tener bronca a alguien. fr. coloq. Am. traer entre ojos. [...]

Ejemplo de uso:

Cara de Ángel, pálido, no puede hablar: tartamudea. Sabe que Colorete le lleva bronca. (30)

Ver también ejemplo de uso en asado, da.

$* * * *$

cebada. [...]\|f. pop. Perú. cerveza.

Agregar esta acepción.

Ejemplos de uso:

Quise darle la mano y decirle: “Te comprendo". Pero qué difícil es sincerarse sin cebada. (23-24)

$* * * *$

Espuma y oro líquido rielan y refulgen en mesas de metal. Radiola loca siete colores, siete maracas. Cubiletes y carajos caen violentos sobre mesas llenas de cebada. (43)

$* * * *$

Siempre he dicho: una mesa, con buenas bandas; un taco, de mi propiedad; tres bolas, sin quiñes; cebada y carretas me bastan para llegar hasta las últimas consecuencias de una vida intensa. (70) $* * * *$

chicoco, ca. [...] m. y f. [...]\| 3. coloq. Perú. Persona que está en la niñez. \| 4. coloq. Perú. Persona que está en la adolescencia.

Agregar estas dos acepciones.

Ejemplos de uso:

Me gustabas como chicoquito pobre, abandonado. Andavete. No vuelvas más por aquí. (62)

$* * * *$ 
-Eres chicoco, todavía, no comprendes. Cuando la vida te golpee, comprenderás que todos los hombres que vivimos "intensamente" guardamos un secreto. (70)

$* * * *$

Juanita, ahora, estás muy cambiada. Pero yo sé que sólo es cáscara. Estoy seguro que basta una palabra mía para que seas la chicoquita de quince años. (83)

Ver también ejemplos de uso en arrochar y sabido.

$* * * *$

collera $^{2}$. [...] || f. coloq. Perú. Grupo de amigos.

Agregar esta acepción.

Ejemplos de uso:

Hace poco no más, los muchachos del billar, la collera del barrio, planearon el robo de una moto. (24)

La collera del barrio, bulliciosa, en tropel (manada de cervatillos montaraces), llega al Paseo de la República. (29)

$* * * *$

La collera, después de discutir el asunto hasta altas horas de la madrugada, se dispersó en la puerta de la Quinta. (50)

$* * * *$

Cuando llegó el verano, con Juanita, con sus amigas y con la collera, me fui a Agua Dulce. (82)

Ver también ejemplo de uso en capazote.

$* * * *$

cuera. [...]\| f. coloq. Perú. Generalmente usando una correa: Castigo.

Agregar esta acepción.

Ejemplo de uso: 
Pero Cara de Ángel me dijo: un día de cuera o todos los días de hambre, escoge. Preferí el día de cuera. (46)

$$
* * * *
$$

desgraciar. [...] || pron. pop. Perú. Volverse asesino.

Agregar esta acepción.

Yo estuve en la sombra, Carambola, pero no por ladrón, sino porque me desgracié. Lo más triste que le puede pasar a un hombre es que lo hagan cojudo. Por eso la maté, Carambola. (71)

gil ${ }^{2}$, la. [...]|| 2. m. y f. pop. Perú. Hombre o mujer. || 3. pop. Perú. Pareja sentimental.

Agregar estas dos acepciones.

Ejemplos de uso:

Autos y tranvías se aglomeran en calles estrechas. Corre, corre apresurado, atropellando gilas, a propósito. (43)

$* * * *$

-Te felicito, Carambola. No hay que perder la ocasión.

-Pero tengo miedo, don Mario: la gila está cerradita. (73)

$* * * *$

¡Pobre Carambola! Si supiera que su tal Alicia es más puta que una gallina. Todas las gilas son igualititas. (76)

Esta noche te abrazo. Te regalo el prendedor. Y te digo despacito: ¿Quieres ser mi gila? (83)

Ver también ejemplos de uso en acostar y pendejo.

lechero, ra. [...] $\|$ adj. coloq. Perú. suertudo. Agregar esta acepción.

Ejemplo de uso: 
Cara de Ángel toma los dados, les echa un poco de saliva y los mueve como si estuviera celebrando culto a una deidad misteriosa, sangrienta. Los deja caer suave; ruedan: marcan diez.

-iQué lechero! (Grita Natkinkón). (34-35)

mandar. [...] $\| \sim$ a pasear a alguien. fr. coloq. Perú. Despedirle con aspereza.

Agregar la forma compleja y la correspondiente definición.

Ejemplo de uso:

Margarita lo mandó, con una palabra deshonesta, a pasear. El Rosquita enfurecido, con bilis, contestó: "Tu boca es parecida a la de esas". (87-88)

$* * * *$

mocoso, sa. [...] || 5. m. y f. Menor de edad.

Agregar esta acepción

Ejemplo de uso:

- ¿No es un poco tarde para ti? Aún eres mocoso y en tu casa te pueden sonar*.

-Yo no soy mocoso y nadies me importa y... además, a nadies le importo en mi casa. (68)

*Ver definición en sonar.

$* * * *$

paleteo. [...] $\| \mathrm{m}$. pop. Perú. Referido a una mujer, generalmente sin su consentimiento: Manoseo de las nalgas.

Agregar esta definición.

Ejemplo de uso:

Bol. Acad. peru. leng. 47(47), 2009 
-Usted que es corrido* sabe que del plan de paleteo y chupete hay que pasar a otra cosa, uno no puede quedarse en el plan de cochineo. $(72-73)$

*Ver definición en corrido.

peluca. [...] || f. pop. Perú. cabello.

Agregar esta acepción.

Ejemplo de uso:

Esta noche no podrá decir que no. Estará alegre. Es su cumpleaños. Y estoy bien firme. Mi peluca está recortada. No hay caso, Manos Voladoras: un artista. Mis zapatos, de gamuza. Estreno pilcha* azul y corbata de seda italiana bien bacán*. (80)

*Ver definición en pilcha y bacán ${ }^{1}$, respectivamente. $* * * *$

pendejo. $[\ldots] \|$... com. coloq. Perú. Persona astuta y taimada.

En este artículo, la entrada debería ser pendejo, ja, ya que en el Perú se distingue el género; por tanto, en vez de com. ('común') se debería colocar la marca gramatical m. y f. ('masculino y femenino'), seguida de la marca de nivel de uso pop. 'popular':

pendejo, ja. [...] ||9. m. y f. pop. Perú. Persona astuta y taimada.

Ejemplo de uso:

Si uno quiere tener amigos y gilas* hay que ser valiente, pendejo. Hay que saber fumar, chupar, jugar, robar, faltar al colegio, sacar plata a maricones y acostarse con putas. (22)

*Ver definición en gil ${ }^{2}$, la. 
polilla. [...]\| f. coloq. despect. Perú. prostituta.

Agregar esta acepción.

Ejemplo de uso:

Y como su casa es estrecha y su hijo duerme en el mismo cuarto y es un estorbo para sus aventuritas, lo manda al taller y mientras mi pobre Príncipe tirita el viejo sucio se revuelca* ${ }^{*}$ calientito con alguna polilla cochina. (42)

*Ver definición en revolcar.

$* * * *$

punto. [...] $\|$ agarrar a alguien de $\sim$. fr. coloq. Arg. Hacerle objeto de bromas. [...]\|t tomar a alguien de $\sim$. fr. coloq. Arg. agarrar de punto. $[\ldots]$

Añadir la marca diatópica Perú en ambas formas complejas y agregar luego la otra forma compleja que se usa aquí con la misma acepción. Quedaría, entonces, de la siguiente manera:

punto. [...] $\|$ agarrar a alguien de $\sim$. fr. coloq. Arg. y Perú. Hacerle objeto de bromas. [...] $\|$ tomar a alguien como . fr. coloq. Perú. agarrar de punto. \|t tomar a alguien de $\sim$. fr. coloq. Arg. y Perú. agarrar de punto. [...]

Ejemplo de uso:

-Ese desgraciado a mí también me tomó como punto -interrumpió Colorete. (45)

sabido. [...] || adj. coloq. Perú. Que sabe aprovechar las circunstancias para lograr determinados fines.

Agregar esta acepción. 
Ejemplo de uso:

Viéndolo bien, ya no eres tan chicoco* que digamos y tienes que ser sabido: a tu edad no sirve amarrarse con hijo. (74)

*Ver definición en chicoco, ca.

$* * * *$

sonar. [...] $\|$ tr. coloq. Perú. Generalmente a correazos: Castigar. Agregar esta acepción.

Ejemplo de uso:

- ¿No es un poco tarde para ti? Aún eres mocoso* y en tu casa te pueden sonar.

-Yo no soy mocoso* y nadies me importa y... además, a nadies le importo en mi casa. (68)

*Ver definición en mocoso, sa.

$* * * *$

sufrido. [...] || estar $\sim$. fr. coloq. Perú. Padecer por estar enamorado. Agregar la forma compleja y la correspondiente definición.

Ejemplo de uso:

En ese momento la odié, la quise ver muerta, muerta; pero, ahora, qué raro, la quiero. No hay caso, estoy sufrido por ella. (60-61)

$* * * *$

taba. [...] || f. pop. Perú. zapato.

Agregar esta acepción.

Ejemplo de uso:

Al día siguiente del asalto, por la mañana, me fui al centro y en Marqueti me compré un pantalón negro, americano, tres casacas bien rocanroleras, dos tabas, como la gran puta, de becerro importado. $(57-58)$

$* * * *$ 
templado. [...]\| adj. coloq. Perú. enamorado ( $\|$ que tiene amor). Agregar esta acepción.

Ejemplo de uso:

-Habla no más, sin miedo, para eso somos hombres.

-Ya, don Mario, pero antes, salud. Este... estoy templado de una chelfa del barrio. (72)

$* * * *$

tono. [...] $\|$ m. coloq. Perú. Fiesta en la que hay música y baile. Agregar esta acepción.

Ejemplo de uso:

Quiero ser como Carambola. O como Natkinkón. Ellos ríen y se alegran con guarachas. En los tonos son de triana. En cambio yo me pongo corto. (79)

Ver también ejemplo de uso en capazote.

$* * * *$

voz. [...]\| pasar la $\sim$. fr. coloq. Perú. avisar $(\|$ dar noticia de un hecho).

Agregar la forma compleja y la correspondiente acepción.

Ejemplo de uso:

-No diga eso, don Mario, me asusta. No se ponga triste; porque yo también me apeno. Si en algo puedo ayudarlo, páseme la voz. (71) $* * * *$

\section{Nuevas entradas y acepciones propuestas al DRAE}

capazote. adj. coloq. Perú. Dicho de una persona: Que es la más capaz.

Bol. Acad. peru. leng. 47(47), 2009 
Ejemplo de uso:

Mi campo es la calle. La collera ${ }^{*} .$. Ahí soy atrevido. En la calle soy el capazote Colorete. Pero en los tonos* me achico. Soy un cobarde. (79) *Ver definición en collera y en tono, respectivamente. $* * * *$

cargamontón. hacer $\sim$. fr. coloq. Perú. Generalmente alrededor de una persona: Arremolinarse alborotadamente.

Ejemplo de uso:

Cara de Ángel se incorpora furioso. Los muchachos ríen y hacen cargamontón. (33)

$* * * *$

enchatar. pron. pop. Perú. emborrachar ( \|causar embriaguez).

Ejemplo de uso:

Caliente me enchaté. Estuve solo en una cantina y toda la tarde puse boleros y guarachas en la radiola. (61)

$* * * *$

ensarte. m. coloq. Perú. fiasco.

Ejemplo de uso:

Hoy me ha pegado. No me quiere. Para ella debo ser ensarte, triple ensarte. (21)

$* * * *$

full. a todo . loc. adv. coloq. Perú. velozmente (\|de manera veloz).

Ejemplo de uso:

Protesté, pero al final, como siempre, se impuso la vieja*. Saqué la bici y, pedaleando a todo full, pasé por la esquina. (22-23)

*Ver definición en viejo, ja. 
planero, ra. adj. pop. Perú. Que acostumbra a tener relaciones amorosas pasajeras.

Ejemplo de uso:

Los muchachos dicen que te has vuelto planera. Pero planera con otros. Con los que no son del barrio. (83)

$* * * *$

tombo. m. pop. Perú. policía ( || miembro del cuerpo encargado de velar por el mantenimiento del orden público).

Ejemplo de uso:

Tranquilo y sereno abrí la puerta. Me senté bien cómodo, como si fuera mío el carro. Encendí el motor y allá me fui, despacito no más, para que el tombo no se diera cuenta. (58)

$* * * *$

trome. com. coloq. Perú. Referido al desempeño de una actividad: Persona que destaca por su destreza. U. t. c. adj.

Ejemplo de uso:

Yo sé que tú quieres ser el trome del billar; pero, para eso, no sólo basta saber manejar el taco. Hay que tener pasión por el juego. (70) $* * * *$

zafa $^{2}$. interj. pop. despect. Perú. U. para echar de un lugar a una persona o animal.

Ejemplo de uso:

Cuando quise entrar, Colorete cogió la bici. Con sonrisa maligna dijo: "Zafa, zafa, no te metas con hombres. Aquí nadies es niñito de casa [...]". (p. 23)

*Ver también ejemplo de uso en espeso.

$* * * *$

Bol. Acad. peru. leng. 47(47), 2009 
De acuerdo con lo expuesto, se puede afirmar que las 47 voces seleccionadas del corpus de la obra Los inocentes responden, en la mayoría de casos, al léxico coloquial y popular del Perú, y que su uso constituye una importante fuente de vitalización de nuestra lengua. Por ello, llama la atención que a 47 años de la primera publicación de la obra de Oswaldo Reynoso, aún no hayan sido consideradas en el DRAE las voces y expresiones propias del habla peruana puestas de manifiesto en Los inocentes y que siguen vigentes hasta el día de hoy. Este hecho resulta bastante preocupante, sobre todo porque todavía hay quienes creen que "una palabra no debe decirse si no está en el diccionario", cuando es evidente que el uso no depende de que el DRAE considere o no una palabra, sino de que ésta se diga y se entienda por quienes la usan, lo que hace, en definitiva, que las palabras mantengan su vigencia.

\section{BIBLIOGRAFÍA}

MARTÍNEZ DE SOUSA, José. Diccionario de lexicografía práctica. Barcelona, Bibliograf S. A., 1995.

PORTILLA DURAND, Luisa P. "Vocabulario diferencial, contrastivo y ejemplificado del español del Perú" en Revista Escritura y Pensamiento N. ${ }^{9}$ 9. Lima, UNMSM, 2002, pp. 139-149. " "Análisis metalexicográfico del Vocabulario de peruanismos de Miguel Ángel Ugarte Chamorro (Lima, 1997)” en Revista Letras N. ${ }^{\text {ss }}$ 105-106. Lima, UNMSM, 2003, pp. 127 140.

"Voces limeñas en el Diccionario de piuranismos de Edmundo Arámbulo Palacios (Piura, 1995)" en Revista Escritura y Pensamiento N. ${ }^{\circ} 16$. Lima, UNMSM, 2005, pp. 213-220.

- "Léxico peruano en la obra En octubre no hay milagros, de Oswaldo Reynoso" en las Actas del I Congreso Internacional de Lexicología y Lexicografía. Lima, APL, 2006, pp. 543-570. 
"Cotejo crítico de las coincidencias léxicas entre Perú y Chile" en las Actas del II Congreso Internacional de Lexicología y Lexicografía. Lima, APL, 2007a, pp. 373-397.

. "Formación del léxico popular limeño: recursos fonéticos, morfológicos y semánticos” en Revista Studium Veritatis N. ${ }^{\text {os }}$ 10-11. Lima, Universidad Católica Sedes Sapientiae, 2007b, pp. 315-354.

. "El DRAE: una tarea que no acaba sino para empezarla de nuevo" en las Actas del III Congreso Internacional de Lexicología y Lexicografía, Lima, APL. En prensa, 2008.

PORTILLA DURAND, Luisa P. et al. Léxico peruano: español de Lima. Lima, APL-USMP, 2008.

REAL ACADEMIA ESPAÑOLA. Diccionario de lengua española. Madrid, Espasa Calpe S. A., 1984.

- Diccionario de lengua española. Madrid, Espasa Calpe S. A., 1992.

. Diccionario de lengua española. Madrid, Espasa Calpe S. A., 2001.

RAMÍREZ, Luis Hernán. "Niveles de uso lingüístico" en Estructura y funcionamiento del lenguaje. Lima, Derrama Magisterial, 7.a edición, 1996, pp. 129-137.

REYNOSO, Oswaldo. Los inocentes. Lima, Peisa, [1961] 1997.

TRUJILLO CARREÑO, Ramón. “El concepto de 'sentido figurado' en el DRAE y cuestiones afines", en Homenaje al Profesor Alvar López. Zaragoza, Institución Fernando el Católico, Excma. Diputación de Zaragoza, 2005, pp. 735-753.

Correspondencia:

Luisa Portilla Durand

Docente de la Facultad de Letras y Ciencias Humanas de la Universidad Nacional Mayor de San Marcos.

Correo electrónico: luisappd@gmail.com

Bol. Acad. peru. leng. 47(47), 2009 